www.jmscr.igmpublication.org

Impact Factor (SJIF): 6.379

Index Copernicus Value: 71.58

ISSN (e)-2347-176x ISSN (p) 2455-0450

crossref DOI: https://dx.doi.org/10.18535/jmscr/v6i5.144

Journal Of Medical Science And Clinical Research

IGM Publication

An Official Publication of IGM Publication

\title{
Open repair of Inguinal Hernia done under Local Anesthesia: Results of one year follow-up
}

\author{
Authors \\ Dr Ajay Kumar Prasad ${ }^{1}$, Dr Hamid Raza Khan ${ }^{2}$ \\ ${ }^{1}$ MGM Medical College and Hospital, Sakchi, Jamshedpur-831001 \\ Address: 33, Kharkai Enclave, Sonari, Jamshedpur-831001 \\ Email: prasadajaykumar938@gmail.com, Contact: 9334777167 \\ ${ }^{2}$ MGM Medical College and Hospital, Sakchi, Jamshedpur-831001 \\ Corresponding Author \\ Dr Hamid Raza Khan \\ Address: H.No.65, Road No.2, B Block, Dhadkidih, P O Bistupur, Jamshedpur - 831001 \\ Email: drhamidrzkhan@gmail.com, Contact: 9431183551
}

\begin{abstract}
Inguinal hernia is a common condition requiring surgical repair, which is conventionally performed under general or regional anesthesia. The area of open repair is usually limited and hence amenable under local anesthesia (LA) too. LA is a safer and has fewer complications, but there is a possibility of intraoperative discomfort to the patient. This study was undertaken to evaluate the efficacy of LA for open repair of inguinal hernia. We studied 72 cases of open surgical repair of inguinal hernia done under LA. All vital parameters were monitored; intraoperative discomfort and the efficacy of repair were evaluated till one year. No patient had any intraoperative complications. $83 \%$ of patients had no intraoperative pain. Average intra op pain was 11 on VAS, and $2.7 \%$ required revision surgery. LA is a safe and effective option in open surgery for repair of inguinal hernia.

Keywords: Open Inguinal hernia repair, local anesthesia.
\end{abstract}

\section{Introduction}

Prevalence of hernia in people above age of 45 years is $4 \%$ and, $75 \%$ of them are inguinal hernias. ${ }^{[1]}$ Surgical repair inguinal hernia is the seventh most common procedures done on human body $^{[2]}$ It is conventionally performed under regional anesthesia (spinal/epidural) and general anesthesia is usually reserved for laparoscopic surgeries. LA for hernia repair was first employed at Lichtenstein hernia institute and Should ice Hospital in the beginning of the last century, and since then, it has been used all over the world. It is the safest option in patients who have multiple comorbidities and are a high risk for any other type of anesthesia. Though it is more economical and reduces the stay in hospital, it is not preferred for the fear of discomfort to the patients and for the concern regarding comfortable operating conditions for the surgeons. There are very few Indian studies regarding hernia repair under LA. This study was undertaken to evaluate the efficacy of LA for the open surgery of inguinal hernia, and its 
impact, if any, on the results of repair, in a medical teaching institute.

\section{Method}

This prospective study was done at the hospital of a medical teaching institute between March 2016 and February 2018 (two years). The study included 72 cases of inguinal hernia repair performed under LA by a team of same anesthetist and general surgeon, with more than 10 years of experience each. Cases of unilateral uncomplicated inguinal hernia who gave consent for the procedure under LA were included in the study. Patients with complex, recurrent, irreducible hernias, uncooperative patients, those opting for laparoscopic procedure and those with obesity (BMI $>30 \mathrm{~kg} / \mathrm{m}^{2}$ ) were excluded. Routinepre-operative investigations were done and written informed consent was obtained. Information sheet was given to the patient and the procedure was explained in details by the principal investigators. An intravenous line was secured. Vital parameters likeECG, pulse rate, oxygen saturation and blood pressure were monitored using a multi-parameter. Intraoperative sedation was given using calculated doses of Fentanyl citrate and Midazolam.

\section{Technique of LA}

Drugs - Anesthetic solution consisted of a 50:50 mixture of $2 \%$ Lignocaine hydrochloride with Adrenaline and $0.5 \%$ Bupivacaine.

Nerve block injections were given for iliohypogastric and Ilioinguinal nerves at a point $2 \mathrm{~cm}$ medial and superior to the anterior superior iliac spine. $10-15 \mathrm{ml}$ of the drug was infiltrated between the planes of external oblique and internal oblique, and that of internal oblique and transversus abdominis.

\section{Local infiltration}

Skin incision was marked by the operating surgeon. $5-7 \mathrm{ml}$ of the prepared mixture was infiltrated into the subdermal region and $3 \mathrm{ml}$ intradermal at the incision site. Subcutaneous infiltration was done at points $2 \mathrm{~cm}$ apart, all along the incision.10-12 $\mathrm{ml}$ of the drug solution was infiltrated below the aponeurosis of external oblique. During the surgery, once the external oblique muscle was opened, the surgeon also infiltrated $10 \mathrm{ml}$ of drug around the nerves, near the pubic tubercle and at the root of the hernial sac near the internal ring.

The hernia was repaired by the appropriate method according to the indication and patient preferences. Eg. Should ice/Bassini technique, Lichtenstein mesh repair etc. Paracetamol 1gm, three to four times a day was administered for post-operative analgesia. Patients were discharged on the next day if there were no complications.

\section{Results}

The mean age of the patients was 62 years. All were males. 32 patients had right sided hernia while 40 patients had left-sided.

48 patients had indirect hernia while 24 patients had direct hernia. The patients were ASA graded between I to III. Hypertension was seen in 20 patients, diabetes mellitus in 14, bronchial asthma in 6 and Ischemic heart disease (IHD) in 7 patients. (Figure No.1)

No intraoperative pain was observed in $83 \%$ of the patients (VAS) while $17 \%$ had mild to moderate grade pain and/or discomfort, which resolved with additional infiltration of the drug locally. (Figure No.2 \& 3) Two patients required intraoperative deep sedation with Propofol. Intraoperative hypotension was seen in one patient.

Operative time (from administration of LA to the end of the procedure) ranged from 45 minutes to 75 minutes with an average of 60 minutes.

There was no post-operative pain in 58 patients, mild pain in 9, moderate in 3 patients and severe in 2, which needed Diclofenac Sodium injection. (Figure No.4)

There were no complications due to the anesthesia or the technique. All the patients were discharged within 24 to 48 hours of the surgery.

Six patients had subcutaneous wound infection. Few stitches were opened and the wound healed 
by second intention. Wound hematoma was observed in 8patients. Two patients required revision surgery within one year of the first repair. (Figure No.5)

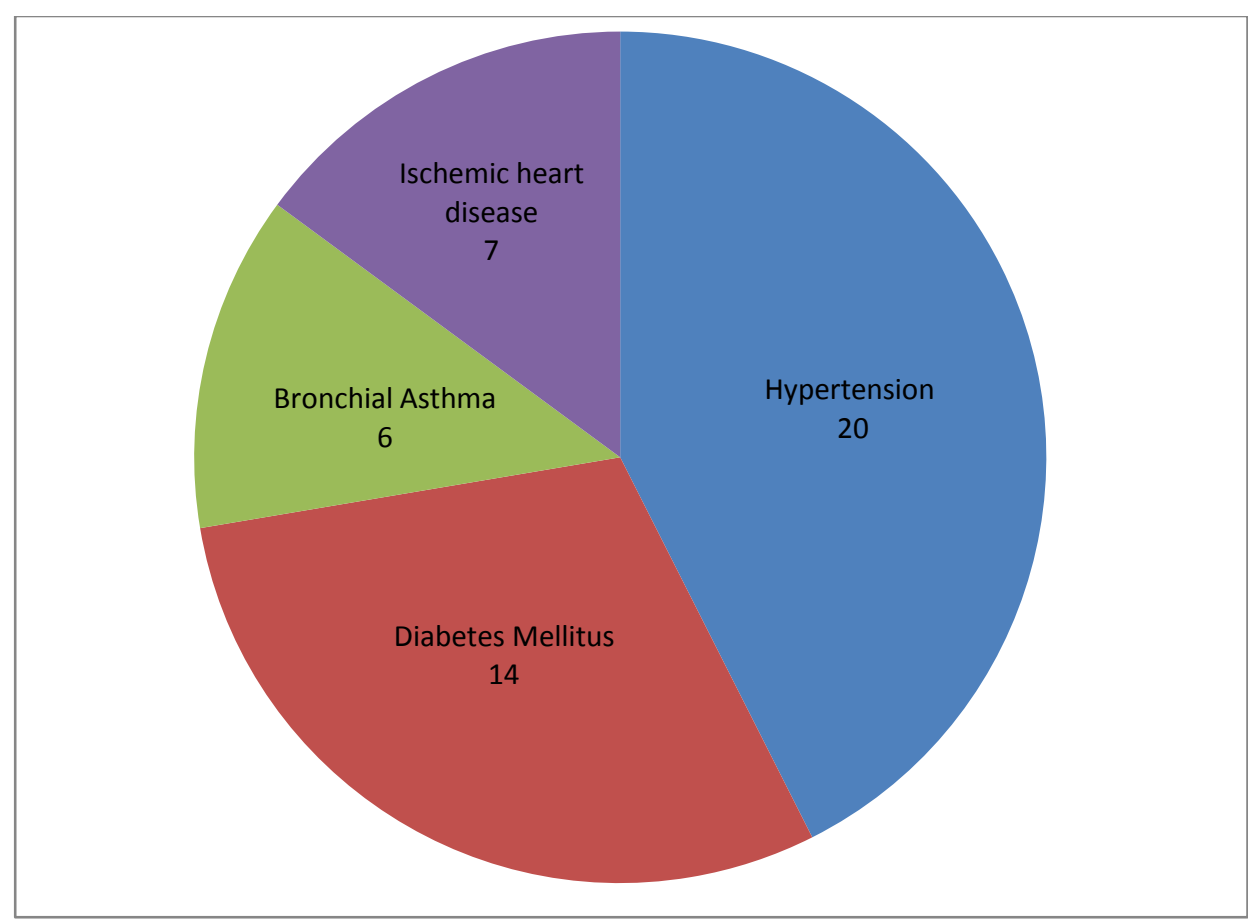

Figure No.1 : Co Morbidities (Total : 47)

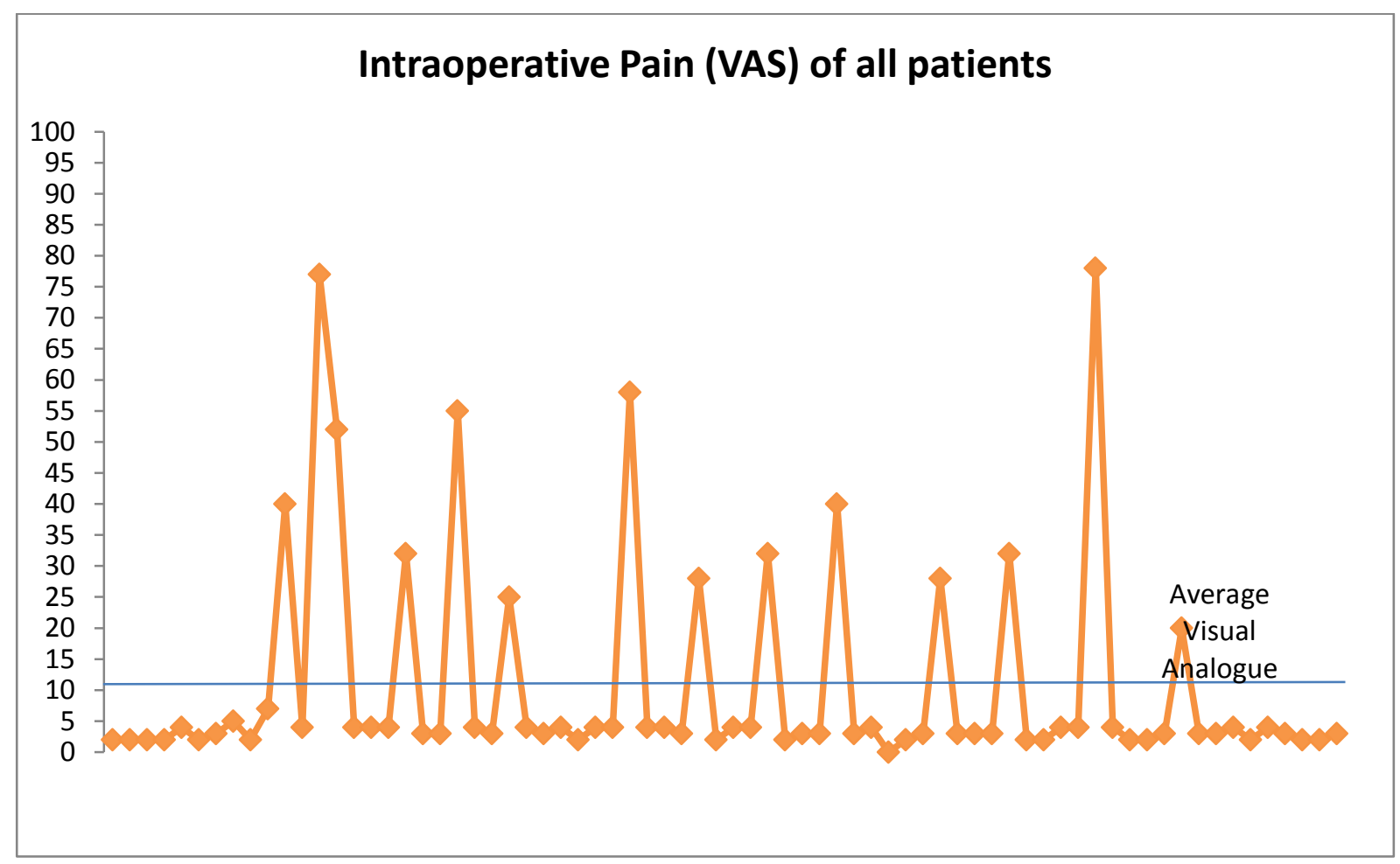

Figure No.2 : VAS scoring of all patients 


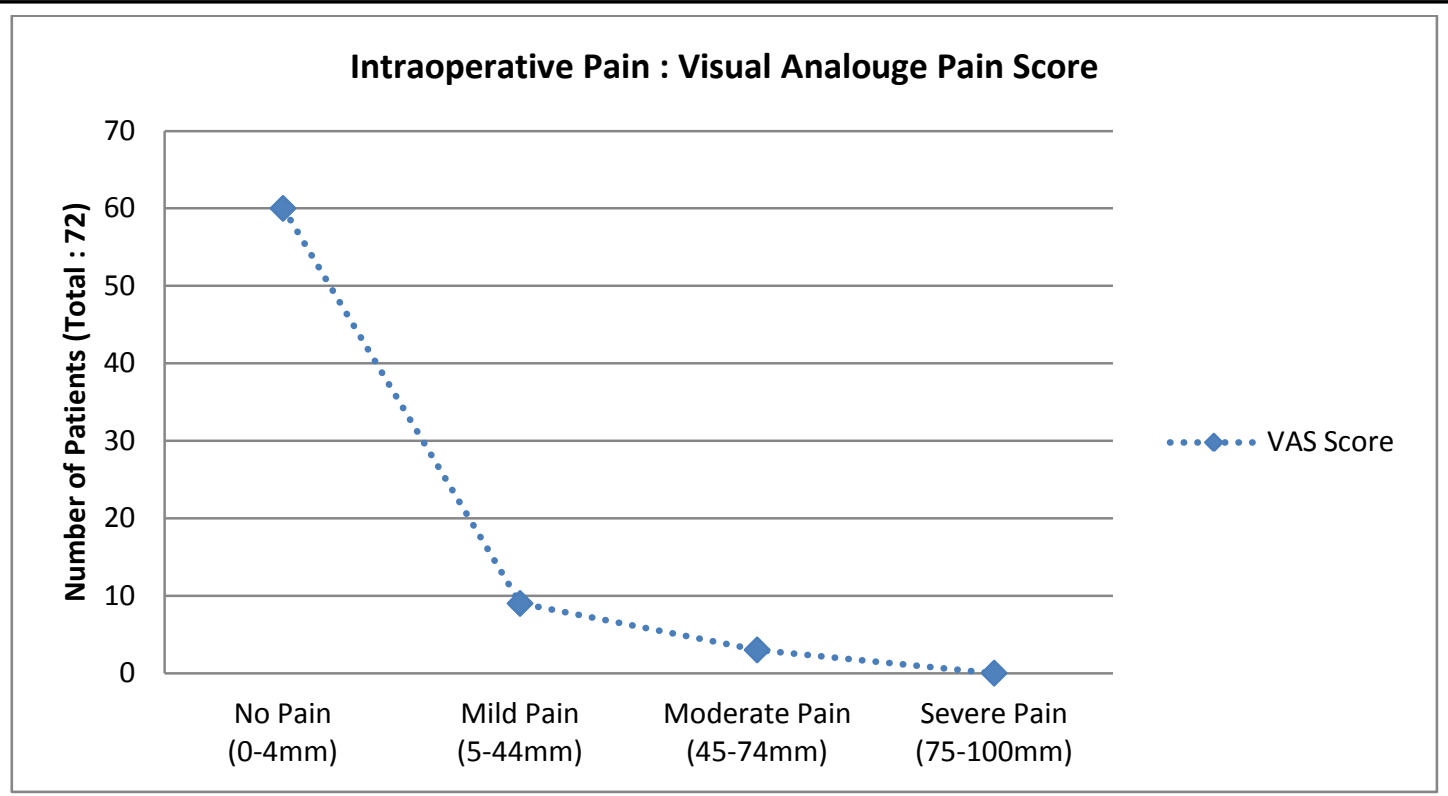

Figure No.3: Intraoperative pain

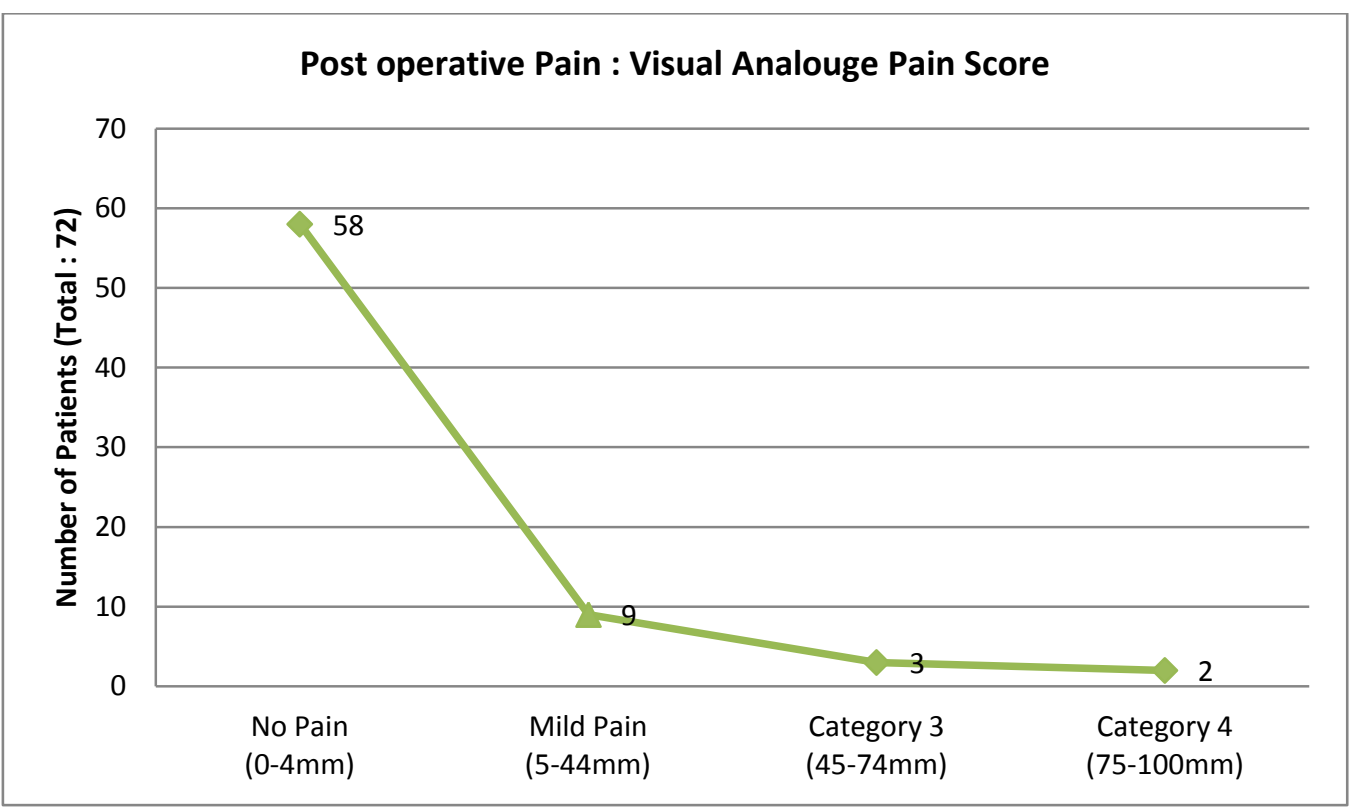

Figure No.4: Post operative pain

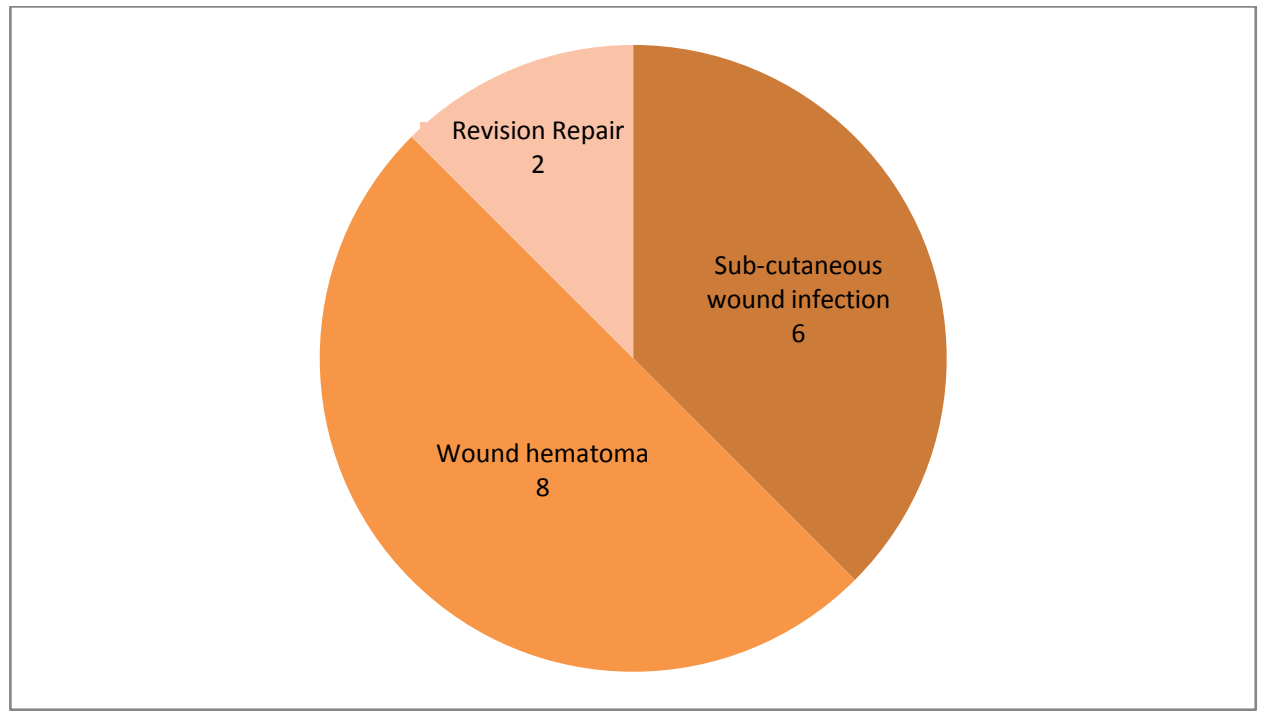

Figure No.5: Post Operative Complications (Total : 16) 


\section{Discussion}

With the advent of better monitoring technology, improved surgical techniques and potent and long acting local anesthetic agents, there is an increasing trend towards local anesthesia in almost all specialties of surgery. Apart of being economical, it is safer and can be used in patients with multiple co-morbities. It has opened surgical possibilities for high risk patients, who are otherwise unfit for any other anesthesia. The use of LA for hernia repair varies in different countries. Eg. in Denmark it is around $18 \%$ and it is almost $100 \%$ in specialized, dedicated institutions like the Should ice clinic and the Toronto clinic. ${ }^{[4]}$ There is apprehension amongst the surgeons as well as patients about the effectiveness of LA for an abdominal surgery. But if it is administered in proper planes, eg. exactlybelow the external oblique aponeurosis, it will result in a successful block. Anderson et al recommended a combination of ilioinguinal block and local infiltration for an effective block. ${ }^{[5]}$

We followed a similar technique in our study. $83 \%$ of the patients experienced no pain during the entire procedure. $17 \%$ of the patients experienced mild to moderate pain during some stage of the surgery, which was managed by further infiltration. Two patients required deep sedation using Propofol. These results were comparable with the study done by Baskerville et al., in which $93 \%$ of the 129 patients operated under LA felt no pain during surgery. ${ }^{[6]}$

It was observed that the two patients which required Propofol, had a large hernia (Gilbert's type $6 \& 7$ ) and there were adhesions of the sac, making the dissection difficult. Hence large hernias may be a contraindication for surgery under LA.

One patient developed hypotension and bradycardia, which may be because of stretching of the peritoneum. ${ }^{[7]}$ It was resolved by releasing the tension on the sac and infiltration of the drug at the inguinal ring.

There was a good post-operative pain relief in, with $81 \%$ patients having no pain after the surgery. $19 \%$ of patients required analgesics in addition to paracetamol. Thus the analgesic dosages required by the patients were less than that is usually required in surgeries under GA/ Regional anesthesia. This is due to the long analgesic effect of local anesthesia, which can be because of the production of nociceptive molecules locally. ${ }^{[8] ~[9] ~}$

2 patients $(2.7 \%)$ presented with recurrence and needed revision surgery under GA/RA. This was found to be consistent with the rates of recurrence in different studies by different techniques, using regional or $\mathrm{GA}$, which is between 0.2 to $10 \%{ }^{[10]}$ [11]

This recurrence depends on various factors like old age, obesity, suture material used, way of dealing with the sac, type of repair done and postoperative complications, apart from the type of anesthesia. ${ }^{[10]}$

A study done by Young et al compared local, regional and general anesthesia for surgery of inguinal hernia. They concluded that the local anesthesia had the least complications and can be done as a day care procedure ${ }^{[12]}$

Most of the patients coming to our institute are from hilly and tribal areas, where transportation is often difficult. Hence we admitted the patients for 24 to 48 hours, to watch for post-operative complications, if any.

The advantages of local anesthesia are that there are no potential complications as with GA/RA like urinary retention, post-spinal headache, hypotension, bradycardia, post-operative nausea \&vomiting.

Certain factors which may affect the dosing of local anesthesia are - age, large hernias, omental mass in the sac, and duration of operation. This was showed by Kulacoqlu et al in their prospective study. ${ }^{[13]}$

Surgical expertise, precise dissection and delicate handling of the soft tissue and an experienced assistant may also be among the factors deciding the efficacy of the LA.

Also, early mobilization and feeding can be started in LA. 
Another advantage of LA is that patient can cough to increase intra-abdominal pressure. This allows the surgeon to evaluate the defect intraoperatively and check the quality of the repair, thus reducing the failure rate.

\section{Conclusion}

Local anesthesia, if properly administered in rightly indicated cases of inguinal hernia is the safest, effective and the most cost effective modality for surgical repair.

It must be advocated and taught more often in medical institutes.

\section{Grants: Nil}

\section{References}

1. Hernias: inguinal and incisional. Kingsnorth A, LeBlanc K, Lancet. 2003 Nov 8; 362(9395):1561-71.

2. An Epidemiological Study of Major Surgical Procedures in an Urban Population of East Delhi Sanjay K. Bhasin et al. Indian J Surg. 2011 Apr; 73(2): 131135

3. Amid PK, Shulman AG, Lichenstein IL. Local anesthesia for inguinal hernia repair step by step procedure. Ann Surg 1994; 220: 735-7.

4. Callesen T. Inguinal hernia repair: anesthesia, pain and convalescence. Dan Med Bull 2003; 50(3):2003-18.

5. Anderson F H, Neilsen k, Kehlet $H$. Combined ilioinguinal blockade and local infiltration anesthesia for groin hernia repair- a double blind randomized study Br.J. Anaesth 2005; 94(4):520-3.

6. Baskerville PA, Jarrett PEM. Day care inguinal hernia repair under local anesthesia. Ann R CollSurgEngl 1983; 65: 224-5.

7. Kulacoglu H, Ozyaylali I, Yazicioqlu D. Factors determining the dose of local anesthetic agent in unilateral inguinal hernia repair. Hernia 2009;13(5) : 511-6.
8. Amid PK, Shulman AG, Lichenstein IL. Local anesthesia for inguinal hernia repair step by step procedure. Ann Surg 1994; 220: 735-7.

9. Callesen T, Bech K, Kehlet H. One thousand consecutive groin hernia repairs under unmonitored local anesthesia .Anesthanalg 2001;93:1373.

10. Shulman AG, Amid PK, Lichtenstein IL. The 'plug' repair of 1402 recurrent inguinal hernias. 20-year experience. Arch Surg. 1990 Feb; 125(2):265-7.

11. Campanelli, Pettinari, Cavalli, Avesani Inguinal hernia recurrence: Classification and approach J Minim Access Surg. 2006 Sep; 2(3): 147-150.

12. Young DV. Comparision of local, spinal and general anesthesia for inguinal hernia repair. Am J Surg 1987;153:560-3.

13. KulacogluH ,Ozyaylali I, Yazicioqlu D. Factors determining the dose of local anesthetic agent in unilateral inguinal hernia repair. Hernia 2009;13(5) : 511-6. 